\title{
Tumoral pulmonary hypertension
}

\author{
Laura C. Price ${ }^{1}$, Michael J. Seckl ${ }^{2}$, Peter Dorfmüller ${ }^{3,4}$ and S. John Wort ${ }^{1}$ \\ Number 2 in the Series "Group 5 Pulmonary Hypertension" \\ Edited by Yochai Adir and Laurent Savale
}

Affiliations: ${ }^{1}$ National Pulmonary Hypertension Service, Royal Brompton Hospital, Imperial College London, London, UK. ${ }^{2}$ Charing Cross Gestational Trophoblastic Disease Centre, Molecular Oncology, CR-UK Laboratories, Hammersmith Hospital Campus of Imperial College London, London, UK. ${ }^{3}$ Pulmonary Vascular

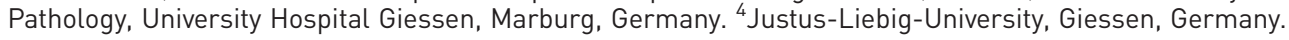

Correspondence: Laura C. Price, Pulmonary Hypertension Service, Royal Brompton Hospital, Sydney Street, London, SW3 6NP, UK. E-mail: laura.pricearbht.nhs.uk

@ERSpublications

Tumoral PH includes pulmonary tumour micro-embolism and pulmonary tumour thrombotic microangiopathy. Diagnosis is difficult and often delayed, with high mortality. Improved survival is reported with some cancer therapies so early recognition is imperative. http://ow.ly/slAn30mLDW7

Cite this article as: Price LC, Seckl MJ, Dorfmüller P, et al. Tumoral pulmonary hypertension. Eur Respir Rev 2019; 28: 180065 [https://doi.org/10.1183/16000617.0065-2018].

ABSTRACT Tumoral pulmonary hypertension (PH) comprises a variety of subtypes in patients with a current or previous malignancy. Tumoral $\mathrm{PH}$ principally includes the tumour-related pulmonary microvascular conditions pulmonary tumour microembolism and pulmonary tumour thrombotic microangiopathy. These inter-related conditions are frequently found in post mortem specimens but are notoriously difficult to diagnose ante mortem. The outlook for patients remains extremely poor although there is some emerging evidence that pulmonary vasodilators and anti-inflammatory approaches may improve survival. Tumoral $\mathrm{PH}$ also includes pulmonary macroembolism and tumours that involve the proximal pulmonary vasculature, such as angiosarcoma; both may mimic pulmonary embolism and chronic thromboembolic PH. Finally, tumoral PH may develop in response to treatments of an underlying malignancy. There is increasing interest in pulmonary arterial hypertension induced by tyrosine kinase inhibitors, such as dasatanib. In addition, radiotherapy and chemotherapeutic agents such as mitomycin-C can cause pulmonary veno-occlusive disease. Tumoral $\mathrm{PH}$ should be considered in any patient presenting with unexplained $\mathrm{PH}$, especially if it is poorly responsive to standard approaches or there is a history of malignancy. This article will describe subtypes of tumoral $\mathrm{PH}$, their pathophysiology, investigation and management options in turn.

\section{Introduction}

Tumoral pulmonary hypertension $(\mathrm{PH})$ comprises a variety of subtypes in patients with a current or previous malignancy. Pulmonary tumour "microvascular disease" includes both pulmonary tumour microembolism (PTE) and pulmonary tumour thrombotic microangiopathy (PTTM). These two conditions are likely to be a disease spectrum. Tumour emboli are frequently reported in autopsy specimens, and may even be asymptomatic. When $\mathrm{PH}$ does develop, progressive and fatal right ventricular failure ensues. Lymphangitic carcinomatosis often coexists but does not usually cause significant $\mathrm{PH}$ in isolation. Tumoral PH can also result from tumour "macroembolism", i.e. the onset of a proximal

Previous articles in this series: No. 1: Jutant E-M, Girerd B, Jaïs X, et al. Pulmonary hypertension associated with neurofibromatosis type 1. Eur Respir Rev 2018; 27: 180053.

Provenance: Commissioned article, peer reviewed.

Received: July 262018 | Accepted after revision: Nov 232018

Copyright CERS 2019. ERR articles are open access and distributed under the terms of the Creative Commons Attribution Non-Commercial Licence 4.0. 
pulmonary embolism related to the tumour-induced prothrombotic environment, with resulting acute right heart failure. The complications of cancer treatments including chemotherapy and radiotherapy as contributors to $\mathrm{PH}$ are increasingly recognised, including cancer treatment-related pulmonary veno-occlusive disease (PVOD). Tumoral $\mathrm{PH}$ is currently classified within group 5 of the clinical classification of $\mathrm{PH}$, reflecting the multifaceted aetiology. This article will describe subtypes of tumoral $\mathrm{PH}$, their pathophysiology, investigation and management options.

\section{Pulmonary microvascular disease}

In the context of tumoral PH, pulmonary microvascular disease comprises PTE and PTTM, which can produce a similar clinical picture to chronic thromboembolic disease. PTE was described in early studies, and defines the occlusion of the pulmonary microvasculature by tumour cells and associated thrombi. PTTM, which was described much later (from 1990) yields a similar clinical picture, and is characterised by more extensive remodelling of pulmonary vessels in association with nests of tumour emboli. These conditions are more commonly a post mortem than ante mortem diagnosis, although PTTM has been increasingly reported ante mortem in recent reports. It is likely that PTE and PTTM represent a disease spectrum related to the degree and vessel location of pulmonary vascular remodelling.

We review the definition, epidemiology and pathophysiology separately; then the clinical presentation, investigations and management of PTE and PTTM together, including recently described cases of PTTM where ante mortem diagnosis provides hope for this devastating condition.

\section{Pulmonary tumour embolism \\ Definition of PTE}

PTE describes the occlusion of small pulmonary arteries by cohesive tumour cells [1], without causing pulmonary metastases [2]. Early descriptions include PTE with "subacute cor pulmonale" in 1937 by Brill and Robertson [1] and, in 1959, BAGSHAwe and BRooks [3] described PTE in a choriocarcinoma patient treated with chemotherapy.

\section{Incidence/epidemiology of PTE}

Post mortem studies suggest that the diagnosis of PTE is greatly underestimated, with autopsy studies showing that $3-26 \%$ of patients with solid tumours had evidence for neoplastic emboli $[2,4,5]$. Therefore, it is likely that a degree of PTE would be an asymptomatic finding in some patients. In the series of mainly carcinoma patients, where WINTERBAUER et al. [5] confirmed PTE in 26\%, 6\% were deemed severe with $>30 \%$ pulmonary arterioles obstructed. In a 1951-1990 retrospective autopsy series of 20 patients with neoplastic emboli, 14 patients were female with a mean (range) age of 49 (18-82) years. Tumour was clinically occult in three patients, and in only one case was PTE and PH clinically considered. The interval between diagnosis of malignancy and development of respiratory symptoms was 14 months, but the mean interval between respiratory symptoms and death was only 1 month [4].

Most reported cases of PTE occur in association with adenocarcinomas, including liver [6, 7], renal, breast $[8,9]$, gastric [5, 7], bladder [1] and choriocarcinoma [3, 10]. In a handful of cases of PTE, estimated at $5 \%$ [11], the primary cancer is unknown.

\section{Pulmonary tumour thrombotic microangiopathy}

PTTM was first described in 1990 by von Herbay et al. [12], in patients with carcinoma. They documented PTTM as a condition that appeared to progress from PTE, from microscopic (not necessarily occlusive) tumour cell emboli to occlusive fibrocellular intimal proliferation, mainly within small pre-capillary pulmonary vessels [12]. Like PTE, PTTM may present as PH of unknown origin, may be difficult to diagnose, and may only be distinguishable from PTE by histological examination. Until recently, PTTM was a post mortem finding with a dismal prognosis $[13,14]$. Recent advances include ante mortem diagnosis and some improvements in short-term outcomes. PTTM has also recently been suggested to be a paraneoplastic syndrome [15]. More recent reports of PTTM, with earlier ante mortem diagnosis, suggest a longer interval between diagnosis and death, but the outlook for these conditions remains dismal.

\section{Definition of PTTM}

PTTM is characterised by the presence of pulmonary vascular tumour microembolic "nests" with evidence for activation of coagulation, obliterative intimal proliferation and ultimately $\mathrm{PH}[2,12]$. PTE is a related condition with cohesive tumour cells but without such marked changes in pulmonary vessel architecture [1]. By definition, pulmonary metastases and larger pulmonary emboli are excluded in these conditions of the pulmonary microcirculation. 
Incidence/epidemiology of PTTM

Like PTE, PTTM typically relates to a carcinoma, usually an adenocarcinoma, including gastric cancer and also breast [16], lung, bladder [17], ovarian clear cell [18], hepatocellular [19], gallbladder carcinoma [20] and choriocarcinoma [21]. A common feature is the presence of additional metastatic disease, often with lymphangitic spread $[18,22]$. An autopsy series of carcinomas suggest that the reported prevalence of PTTM is $1-3 \%[22,23]$. Most reported cases of PTTM have been described in Japan, likely reflecting their high prevalence of gastric adenocarcinoma. PTTM occurs in 16-27\% of cases of gastric carcinoma [14, 22, 24], especially the mucinous, signet ring and poorly differentiated subtypes $[13,22,24]$

\section{Pathogenesis of PTE/PTTM}

As previously stated, small vessel PTE are observed in autopsy specimens in much higher numbers than are clinically apparent $[2,4,5]$. In terms of how and why tumour cells lodge in the pulmonary vessels, it is first important to consider that all tumours metastasise haematogeneously to the pulmonary circulation, especially lung, thyroid, renal and liver cancers. This relates to direct venous extension of tumour cells or fragments, as can also occur via the subhepatic veins in hepatic metastases from any primary [6].

In the 1951-1990 autopsy series of 20 patients with neoplastic emboli mentioned above, SHIELDS and EDWARDS [4] identified three subgroups based on microscopic and gross features: 1) six patients with predominantly neoplastic microemboli (PTE) (figure 1a); 2) 10 patients with mixed neoplastic and thrombotic microemboli (later termed PTTM) (figure $1 \mathrm{~b}$ and c); and 3) four cases with both neoplastic microemboli and large, fatal tumour macroemboli (primary tumours in kidney, femur, cervix and thyroid). They noted changes in the morphology of resistance vessels of the lung (small arteries and arterioles), including pulmonary arterial hypertension (PAH)-like medial and intimal hypertrophy, intimal fibrosis and fibrinoid necrosis of the vessel wall, as well as noticing that plexiform lesions were notably absent. Medial hypertrophy was grade 1 (11-15\% remodelling of the smooth muscle layer) in 12 cases, grade $2(16-25 \%)$ in two cases and normal grade $0(<10 \%)$ in six cases. This is indicative of the subacute nature of the disease, although no cases described severe grade 3 (>25\%) remodelling [4].

In more recently described cases of PTTM, there appears to be an intimate association between nests of tumour cells and surrounding fibrointimal proliferation in pulmonary arteries and veins, which ultimately leads to vessel occlusion (figure $2 \mathrm{a}$ and $\mathrm{b}$ ). The proliferating intimal cells are both endothelial and non-endothelial cell (i.e. myofibroblastic) in origin [22]. Cancer cell attachment is postulated to cause intimal (endothelial cell) damage, activation of coagulation and initiation of proliferation through growth factors and cytokine release/expression, including tissue factor, vascular endothelial growth factor (VEGF) $[14,23,24]$ and platelet-derived growth factor (PDGF) $[24,25]$. PDGF is known to initiate macrophage recruitment and upregulate VEGF on cancer cells, which may further increase endothelial cell proliferation. Interestingly, imatinib (through c-KIT as well as PDGF inhibition) led to re-canalisation of previously occluded small pulmonary arteries in a case of PTTM related to gastric cancer [25].

Vascular inflammation is also likely to contribute to the pathogenesis of PTTM [26]. Dense perivascular and intimal accumulation of macrophages have been reported $[21,27,28]$ (figure 2b). Putative mediators

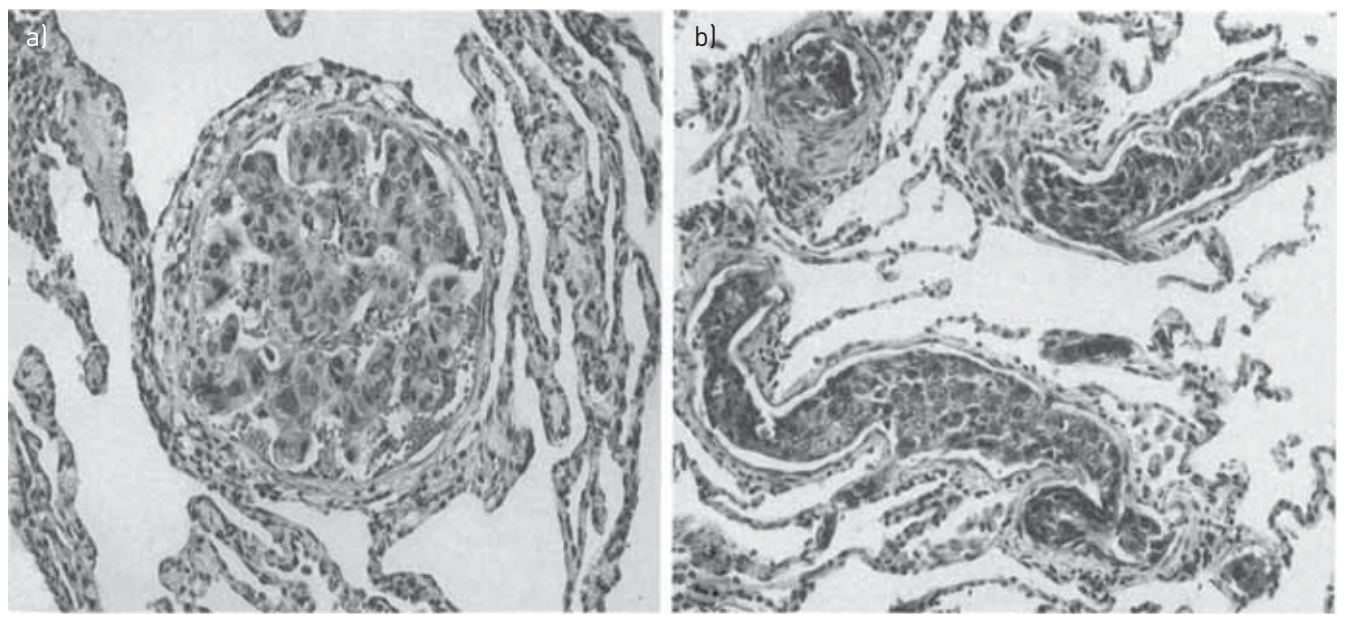

FIGURE 1 Neoplastic emboli. Photomicrographs of neoplastic pulmonary emboli. a) Occlusion of a small muscular pulmonary artery by tumour cells from a 55-year-old woman with breast carcinoma. b) Obstruction of multiple pulmonary arterioles by tumour cells from a 36-year-old woman with cervical squamous-cell carcinoma. (Haematoxylin and eosin stain $\times 120$ ). Reproduced from [4]. 

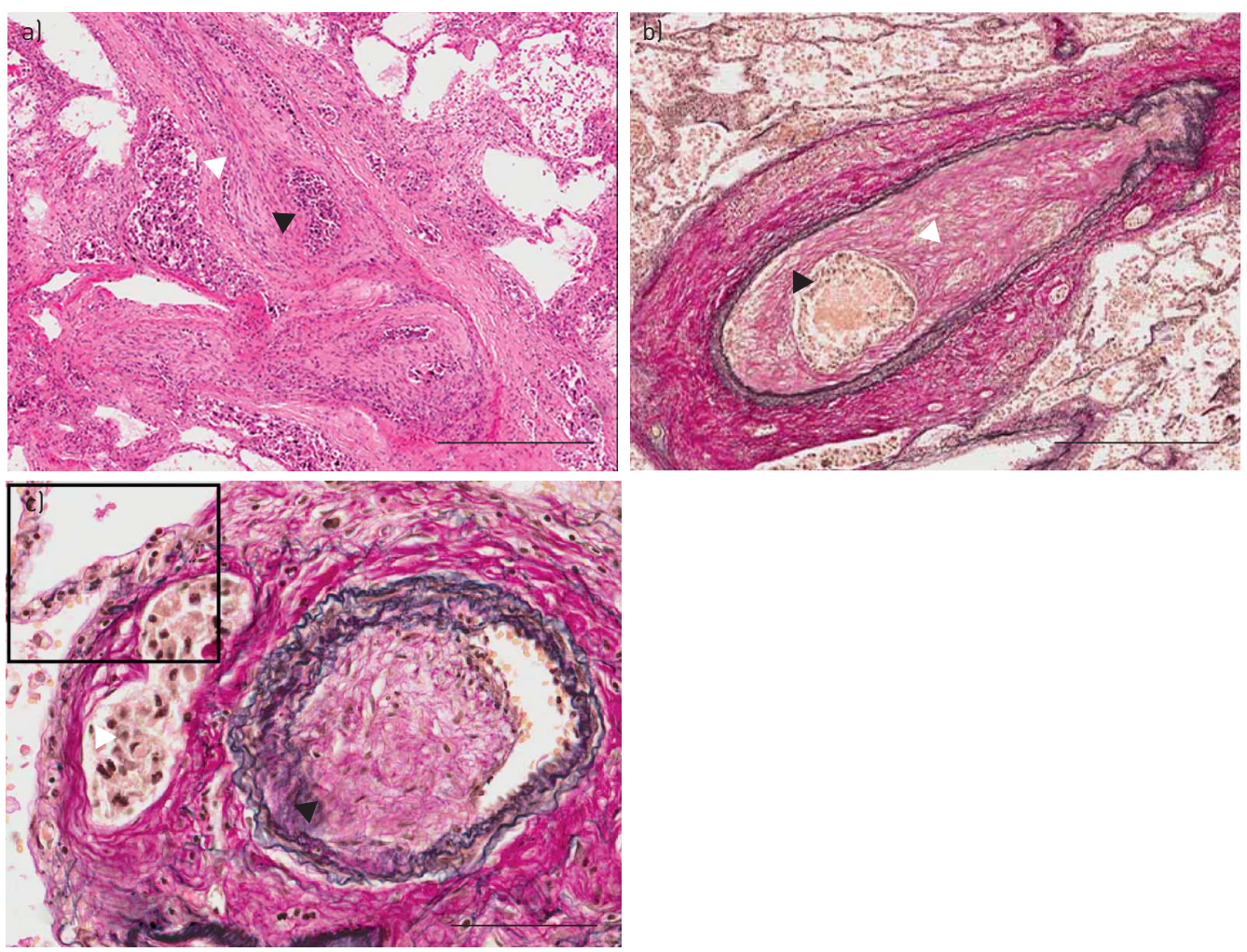

FIGURE 2 Lung histology from a case of pulmonary tumour thrombotic microangiopathy related to severe pulmonary hypertension. a) Post mortem section showing occlusion of a medium-sized pulmonary arterial lumen by fibrointimal proliferation of fibroblasts and collagen (white arrow) and tumour emboli (black arrow) (Haematoxylin and eosin stain $\times 4.3$ ). Scale bar=500 $\mu \mathrm{m}$. b) Post mortem section showing a medium-sized pulmonary artery with two elastic layers, with a normal-sized smooth muscle layer. There is exaggerated luminal occlusion by fibrointimal thickening (white arrow) surrounding nests of tumour emboli (black arrow). The adventitia contains lymphatic tumoral thrombi. Increased alveolar macrophages are seen surrounding the lung (Elastica van Gieson stain $\times 4.6$ ). Scale bar $=500 \mu \mathrm{m}$. c) Evidence for fibrointimal proliferation within the lumen of small pulmonary veins (black arrow) and tumour involvement of accompanying lymphatics (white arrow). Inset: veins close to the centrilobular bronchovascular bundles show eccentric fibrointimal remodelling (Elastica van Gieson ×28.4). Scale bar=80 $\mu \mathrm{m}$. Reproduced from [26].

include osteopontin, a cytokine and adhesive protein that is implicated in tumoral thrombosis and neointima formation [28], as well as promotion of progression and metastasis of cancer [29]. In PTTM, macrophages stain for osteopontin and CD44 (an osteopontin receptor) [28], with tumour cells and proliferating fibrointimal cells also overexpressing both CD44 and osteopontin, as well as PDGF and VEGF $[27,28]$. Therefore, crosstalk between tumour cells, macrophages and intimal cells may occur through the osteopontin-CD44 axis to drive both tumorigenesis and ongoing macrophage recruitment. Macrophage-derived factors, such as interleukin-6, as implicated in PAH [30], may also contribute to the fibrointimal proliferation in PTTM.

Tumour cell nests are also evident in pulmonary veins and lymphatics [16, 21, 24], with evidence for lymphatic tumour invasion (figure 2c). Fibrointimal proliferation in pulmonary veins in PTTM lesions has been reported in several recent reports [21,31], and may be analogous to the remodelling of pulmonary veins seen in distal chronic thromboembolic PH (CTEPH) [32]. Putative interactions between tumour cells, macrophages and vascular cells are summarised in figure 3.

\section{Development of $\mathrm{PH}$}

There are two hypotheses for the development of PH in PTE/PTTM. First, inadequate clearance of occlusive tumour emboli may increase pulmonary vascular resistance through mechanical obstruction. In PTE, a median $30 \%$ of vessels were occluded in symptomatic patients [2]. PH will develop in PTTM as progressively more vessels become stenotic and occluded by the fibrointimal layer [13, 22]. Analysis of autopsy specimens from patients with PTTM showed variable luminal occlusion but that widespread severe luminal narrowing was only a feature in severe $\mathrm{PH}$ [24].

Secondly, vascular occlusion is also likely to relate to PAH-like pulmonary vascular remodelling. There are many unanswered questions in terms of signalling, including the potential role of genetic influences in 


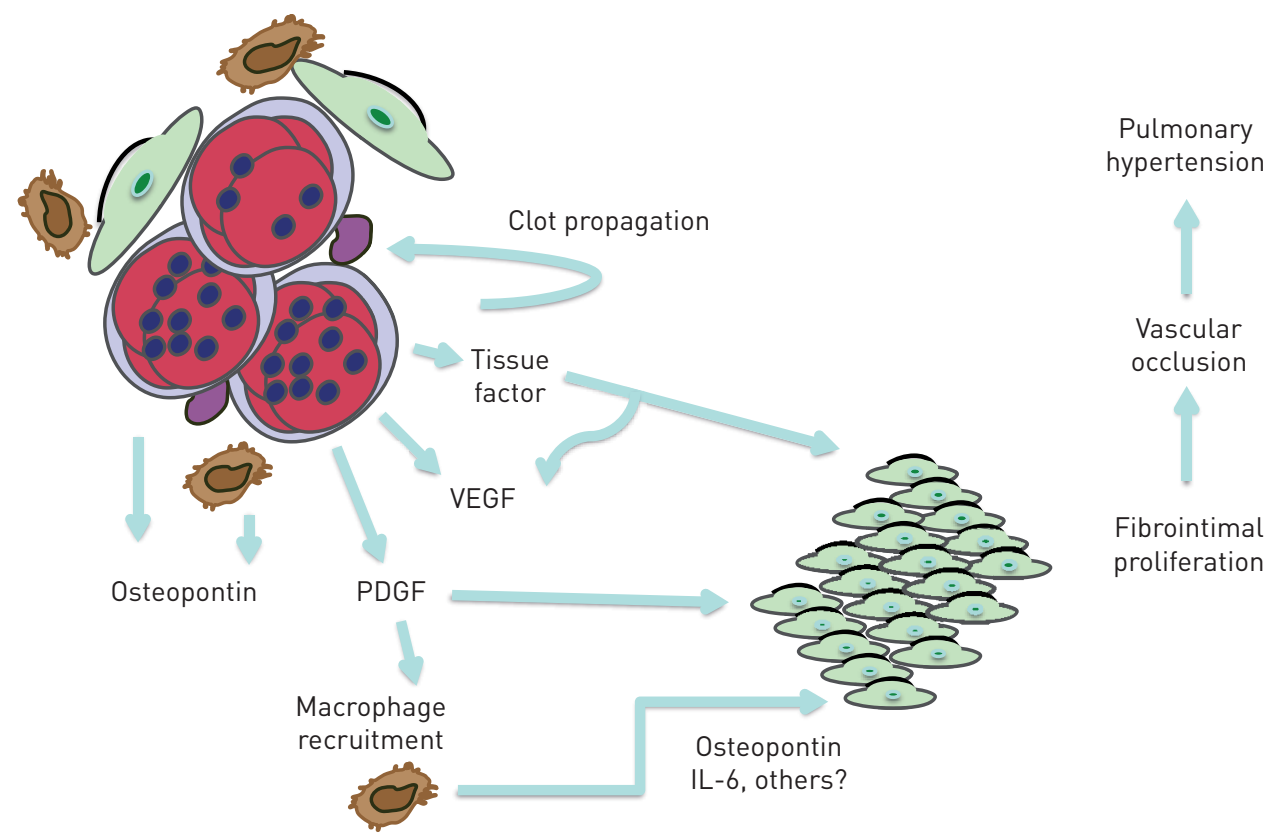

Carcinoma cell $\bigcirc$ Platelet $\rightleftharpoons$ Endothelial/intimal cell

FIGURE 3 Proposed mechanisms for fibrointimal proliferation in pulmonary tumour thrombotic microangiopathy (PTTM). Small nests of carcinomatous cells lodge in the pulmonary vessels, including small pre-capillary arteries (via haematogenous spread) and on the post-capillary side of the pulmonary circulation to pulmonary veins and lymphatics (by lymphatic invasion). Tumour cell and endothelial cell interaction initiates clot formation, and releases further cytokines including vascular endothelial growth factor (VEGF) and platelet-derived growth factor (PDGF). This initiates macrophage recruitment and intimal lendothelial cell and non-endothelial cell like, i.e. myofibroblastic) proliferation. Tissue factor also upregulates VEGF expression on tumour cells, which is angiogenic to intimal cells. PDGF-A and -B are expressed on tumour cells, and anti-phospho-PDGFR- $\alpha$ is expressed on vascular endothelial cell and gastric carcinoma cells. This indicates that PDGF signalling activation of tumour cell growth is present through both autocrine and paracrine mechanisms. The cytokine and adhesive protein osteopontin is expressed on tumour cells in PTTM, and is likely to be a key driver for intimal cell growth. Perivascular CD68-positive macrophages are noted and also reside within intimal layers. Macrophages also stain for CD44, the adhesion molecule which interacts with osteopontin to induce chemotaxis of T-cells and macrophages, propagation of local inflammation and intimal proliferation (through other known macrophage-derived pro-proliferative factors including interleukin (IL)-6). Direct contact with tumour nests is not universal in all vessels where remodelling is present.

terms of why a large number of patients have asymptomatic tumour emboli but a very small number progress to PTE or PTTM.

\section{Haematological aspects}

The onset of venous thromboembolism in patients with cancer is of course well described. For example in patients with acute pulmonary embolism, where an underlying malignancy should be excluded, and also in patients with CTEPH. Carcinomas promote coagulation by several mechanisms [33], such as the release of microvesicles containing tissue factor [24] and through interaction (by mucinous carcinomas) with selectin on platelets [34]. Binding of tissue factor with factor VII and calcium ultimately leads to fibrin deposition and platelet activation to form clots. Progressive disseminated intravascular coagulation consumes fibrin, with release of fibrinogen degradation products or d-dimers. Usually, disseminated intravascular coagulation is rapid and associated with bleeding, whereas in malignancy "compensated disseminated intravascular coagulation" can occur, where the rate of consumption of coagulation factors and platelets is slow; thrombotic manifestations exceed bleeding events [35]. Evidence for raised d-dimer or fibrinogen degradation products has been a uniform finding when reported in cases of PTTM [23,35], with features of microangiopathic haemolytic anaemia (MAHA) or disseminated intravascular coagulation in half of cases at presentation [23, 35-37].

\section{PH classification of PTE/PTTM}

PTE was first classified in 2004 within group 4 of the World Health Organization (WHO) classification system of $\mathrm{PH}$ [38], and in the most recent update "other intravascular tumours" remains within group 4.2.2 [39]. 
PTTM, with increasing recognition and understanding of its multifaceted mechanisms, now lies within group 5, i.e. that described to reflect multifactorial and/or unclear mechanisms. Tumoral obstruction, therefore, sits within subgroup 5.4 of the current classification of $\mathrm{PH}$ [39]. However, this classification does not necessarily guide management.

\section{Clinical presentation of PTE/PTTM}

Patients with PTTM usually present with progressive exertional breathlessness, hypoxia and progression right ventricular (RV) dysfunction [23]. Onset is usually 3 weeks to 6 months prior to presentation [14, 22, 40]. Cough may predate dyspnoea. Haemoptysis, chest and abdominal pain, which may reflect liver metastases or venous congestion, have also been reported [37]. The progression of symptoms correlates with high-resolution computed tomography (HRCT) findings prior to the onset of $\mathrm{PH}$, which may be a few weeks to months later [40]. Fatigue is also reported [41]. SOAREs et al. [6] compared symptoms in a group of pathologically confirmed tumour emboli patients with lymphangitic patients. The tumour embolism group was more likely to have dyspnoea (58\%) than those with lymphatic disease (46\%), however diagnostic utility of this symptom was low [6].

Depending on the timing of presentation, symptoms and signs, $\mathrm{PH}$ may or may not be present. These include a prominent pulmonary second heart sound, raised jugular venous pressure, a right parasternal heave and further signs of RV decompensation. However these signs may often be absent even with confirmed $\mathrm{PH}[42]$.

\section{Investigations for PTE/PTTM}

Diagnosis of PTTM can be challenging and many investigative findings are nonspecific. ECG may show signs of RV strain in the presence of $\mathrm{PH}$ including an S1Q3T3 appearance. Chest radiography is often normal. Bloods demonstrate raised d-dimer or fibrinogen degradation products [23] and, in some cases, MAHA. Patients desaturate markedly on 6-min walk test assessment. Pulmonary function testing would show pulmonary vascular limitation, characterised by relative preservation of lung volumes but very low gas transfer values (transfer factor of the lung for carbon monoxide 25-30\%), even lower than in PAH [43].

Radionucleotide ventilation/perfusion (V/Q) scanning may show multiple small peripheral sub-segmental perfusion defects not evident on computed tomography pulmonary angiography, with normal ventilation (figure 4a). However, V/Q scanning abnormalities do not reliably distinguish PVOD from idiopathic PAH $[4,44]$. Serial V/Q may show resolution of perfusion defects following treatment [25].

The confirmation of $\mathrm{PH}$, once suspected clinically and suggested by a raised brain natriuretic peptide with echocardiographic findings, is by right heart catheterisation. Pre-capillary $\mathrm{PH}$ is defined by a mean pulmonary artery pressure $\geqslant 25 \mathrm{mmHg}$ and pulmonary capillary wedge pressure $<15 \mathrm{mmHg}$ [39]. The assessment of haemodynamic severity would be consistent with all other causes of $\mathrm{PH}$, with signs of right heart dysfunction and low cardiac index being key prognostic factors [39]. At the time of right heart catheterisation, pulmonary wedge aspiration cytology should be performed to detect tumour cells (see below) to attempt to confirm the diagnosis of PTTM or PTE.

\section{Radiological findings in PTE/PTTM}

Plain chest radiography is often normal or shows diffuse reticulonodular opacities, and less often Kerley B lines and pleural effusions [45]. Parenchymal computed tomography (CT) abnormalities (figure 4b) are nonspecific and include centrilobular nodules, ground-glass attenuation, interlobular septal thickening and consolidation [23].

CT signs of PH may be present including enlargement of the central pulmonary artery, right heart chamber enlargement and flattening of the intraventricular septum [21, 45]. Other pulmonary manifestations of malignancy may be present, such as discrete metastatic deposits.

\section{Main HRCT findings}

Compared to PTE, where HRCT appearances are more often unremarkable, HRCT in PTTM may show centrilobular nodularity, ground-glass opacities and interlobular septal thickening (figure $4 \mathrm{~b}$ ). These CT appearances will be discussed in turn.

Centrilobular nodularity on HRCT describes a central small nodule within the secondary pulmonary lobule, and is often reported in PTTM [21, 40,41]. The nodularity is usually of an ultrafine granular appearance and is likely to represent the peripheral pulmonary arterial lesions. These opacities are usually well defined as they reflect peripheral blood vessels rather than the respiratory bronchioles when the nodule border is usually more blurred, for example in bronchiolitis. Centrilobular nodularity on HRCT is an early sign in PTTM, and may disappear following chemotherapy [40], in keeping with resolution of the 
a)
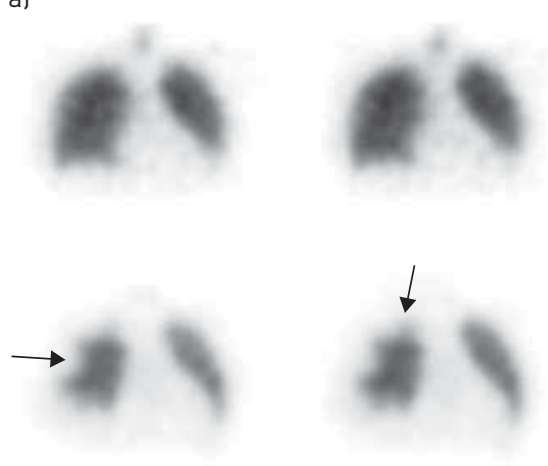

VENTILATION

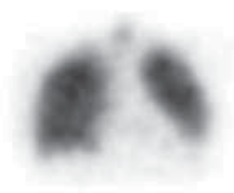

PERFUSION

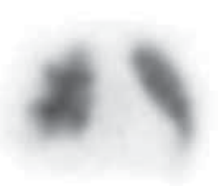

FIGURE 4 Radiology in pulmonary tumour thrombotic microangiopathy (PTTM). a) Ventilation/perfusion demonstrating sub-segmental defects in lung perfusion in a patient with PTTM. Computed tomography pulmonary angiography did not demonstrate these peripheral lesions (not shown). b) High-resolution computed tomography scanning in PTTM showing widespread groundglass opacification, micronodules, interlobular septal thickening and small bilateral pleural effusions.

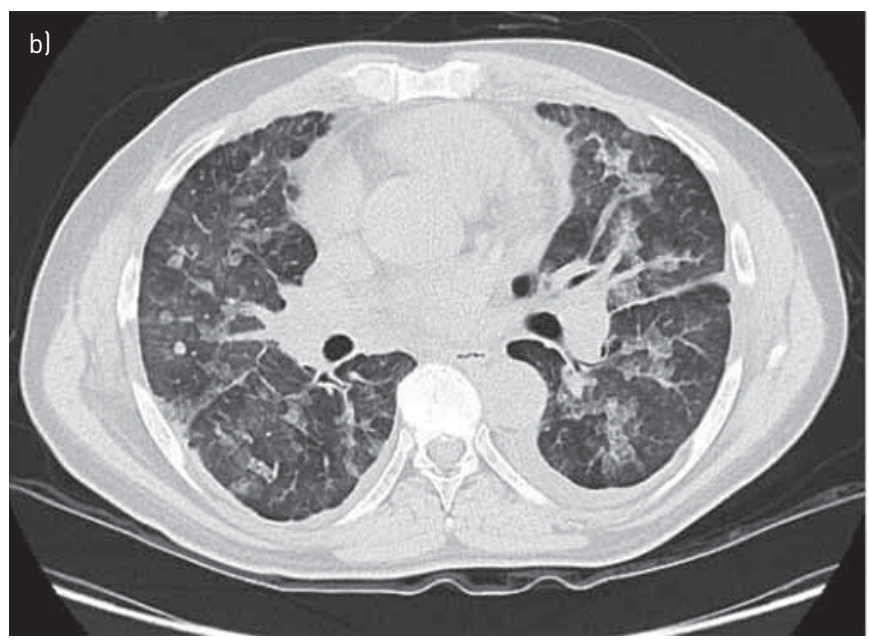

small vessel lesions. Quite frequently the centrilobular nodules exhibit a tree in bud nodular pattern, a feature usually associated with plugging of respiratory bronchioles rather than vascular disease [46].

Ground-glass opacities are reported in PTTM (often patchy or wedged shaped) [21,41,47], for which the radiological differential diagnosis remains broad. In the setting of PTTM and PH, especially with postcapillary involvement, ground-glass opacities may be consistent with interstitial and airspace oedema [21], or interstitial inflammation, as suggested by resolution 1 month after high-dose steroids [28].

Interlobular septal thickening on HRCT (the corollary to septal lines on chest radiography) is described in PTTM, and is again a nonspecific finding. It simply indicates disease of the connective tissue, lymphatics and/or pulmonary veins. The nature and distribution of septal thickening may provide clues to aetiology. In PTTM it appears smooth and peripheral in distribution [21,41,48], usually without pleural effusions. Lymphangitis carcinomatosa tends to cause more irregular, nodular interlobular septal thickening, resulting in prominence of the secondary pulmonary nodules, as well as thickening of the bronchovascular interstitium, subpleural nodules, pleural effusions and often hilar or mediastinal node enlargement. Distinguishing PTTM from lymphangitis carcinomatosa may be difficult and of course these conditions may coexist.

\section{Further assessment for malignancy}

Investigations in a patient with new-onset PH and suspected PTE/PTTM should include screening for undiagnosed malignancy. This may involve a full clinical assessment similar to the assessment of unprovoked pulmonary embolism [49]: mammography, a contrast-enhanced CT abdomen/pelvis and consideration of further targeted tests [25]. This has formed part of our usual practice given the increased awareness of PTTM, although it is not specified in current PH guidelines [39]. Raised tumour markers such as CEA, CA19-9, CA15-3 and SCC may provide clues but are not diagnostic of an underlying cancer [40], except for human chorionic gonadotrophin and alpha feto-protein. The former in the absence of pregnancy or false-positive readings is diagnostic of gestational and non-gestational cancers and the latter is highly indicative of germ cell and hepatocellular cancers. If the patient is too ill for a biopsy of a suspicious lung cancer then venous blood sampling to request mutant epidermal growth factor receptor (EGFR) testing on extracted circulating free DNA can diagnose an EGFR driven lung adenocarcinoma. This enables commencement of life-saving therapy with EGFR targeted drugs, such as erlotinib or 
gefinitib [50]. Liquid biopsies (cell-free DNA) can also be performed for other cancer types, such as breast and melanoma.

\section{Pulmonary wedge aspiration cytology and lung biopsy}

The diagnosis of PTE/PTTM can be made by the cytological examination of the aspirate from a wedged pulmonary artery catheter [1]. This technique has a reported sensitivity of $80-88 \%$ and a specificity of $82-$ $94 \%[5,6]$. It is reported in both PTTM and PTE [51], but it would not be possible to distinguish between these. Of note, if the sample is not wedged, malignant cells from "upstream" (i.e. the liver) may contaminate the sample [1]. The sample should be heparinised, centrifuged and the buffy coat examined for malignant cells. Lung biopsy has been used to diagnose PTTM ante mortem either guided by CT, bronchoscopically or using video-assisted thoracoscopic surgery [37, 40, 47]. However, we would advise caution in the presence of $\mathrm{PH}$.

\section{PET in PTTM}

The use of 2-(F-18)-fluoro-2-deoxy-D-glucose positron emission tomography (FDG-PET) has been reported in PTTM $[23,36,48]$ and, for example, showed uptake in areas of primary lung cancer and consolidation pathologically proven to be PTTM [23]. However, PTTM may be PET negative [40], which may relate to size of lesions or that certain histological subtypes of gastric adenocarcinoma are less FDG-PET avid [52].

\section{Management of PTE/PTTM}

Until recently, all case reports reported death soon after diagnosis. Unsurprisingly, anecdotal reports of therapeutic thrombolysis have shown no benefit [53]. Ante mortem diagnosis has enabled more aggressive treatment of the underlying cancer in both PTE and PTTM (using chemotherapy or targeted agents) and, potentially, the PTTM (using anti-proliferative approaches). In some cases a combined approach has been used for periods up to 14 months, alongside basic therapies including anticoagulation and oxygen therapy [47]. The role for conventional pulmonary vasodilators is uncertain. Current evidence is based purely on reported cases.

\section{Pulmonary vasodilators}

Although no evidence suggests that the usual vasoactive mediators implicated in PAH are anti-remodelling targets in PTE/PTTM, there is rationale for their use in abating pulmonary vasoconstriction. Endothelin receptor antagonists including bosentan [41] and ambrisentan [21, 25] have recently been used in cases of PTTM. In some cases, PTTM progressed despite their use [21, 28, 41]. In other cases, PTTM improved when pulmonary vasodilators were used in combination with imatinib [25, 41].

\section{Chemotherapy}

The reduction in the number of malignant cells by chemotherapy might lessen the stimulus to fibrointimal proliferation. Reports suggest that early use is effective in that it may resolve cough and centrilobular nodules on HRCT [40]. A combination approach, for example with dexamethasone, warfarin and aspirin [37], may be more beneficial, with therapeutic response seen with falling d-dimer, serum VEGF and tumour markers $[40,47,54]$. Efficacy is likely to depend on chemosensitivity [55].

\section{Anti-inflammatory/antiproliferative approaches}

These include broad "anti-inflammatory" inhibition using glucocorticoids or the specific blockade of relevant growth factors. The use of dexamethasone $\left(0.05 \mathrm{mg} \cdot \mathrm{kg}^{-1}\right.$ daily, in combination with anticoagulation and chemotherapy) resulted in permanent resolution in one case of gastric cancer-associated PTTM [37], and to very short-term improvement in HRCT changes, cough, hypoxia and haemodynamics in a patient with recurrent breast carcinoma-associated-PTTM [28].

The tyrosine kinase inhibitor imatinib blocks phosphorylation of the PDGF receptor, and inhibits downstream cell growth. It is approved as an anticancer drug. Immunohistochemical studies support a basis for blockade of PDGF in PTTM [14, 23, 24]. PDGF signalling is also implicated in PAH [56], although clinical studies of imatinib in PAH have been complicated by adverse events including subdural haematomas in patients receiving anticoagulation [57]. Several reports suggest that imatinib may extend survival in PTTM $[25,41]$, where imatinib led to reduced serum PDGF-BB and brain natriuretic peptide levels alongside perfusion defects on $\mathrm{V} / \mathrm{Q}$, with persistent normalisation of invasive pulmonary haemodynamics. Although $\mathrm{PH}$ did not recur, both patients died of non-PH systemic metastatic complications at 10-12 months [25, 41]. Further to PDGF inhibition, VEGF inhibition using bevacizumab alone then in combination with imatinib was effective in treating $\mathrm{PH}$ with an associated fall in serum 
VEGF levels. Again, the patient died of a non-PH cause at 12 months [58]. These cases targeting specific growth factors are encouraging and require systematic assessment.

\section{Tumour macroembolism}

Rarely, a large tumour metastasis can mimic a proximal pulmonary embolism. Clinical features including rapid onset of symptoms with $\mathrm{PH}$ and acute right heart failure would be indistinguishable. Tumour macroembolism is reported in cases of breast cancer [59], hepatocellular carcinoma [60], choriocarcinoma $[10,55,61,62]$ and, less often, subdiaphragmatic cancers including cervical carcinoma [63]. PTE/PTTM may of course coexist $[4,60]$. These reflect the tumours that more commonly spread to the heart and great vessels (renal, lung, breast, oesophagus, malignant lymphoma, leukaemia and malignant melanoma), through the vena cava (including invasive renal tumours that may fragment), arterial circulation, retrograde lymph node spread, or directly from adjacent viscera [63]. Overall metastatic cardiac cancer rates are low at $1.2 \%$ [64], and thought to relate to cardiac movement thereby rapid blood and lymphatic flow away from the heart [63]. In addition, some tumours may compress the proximal pulmonary arteries, with or without additional lymph nodal compression.

Finally, it should be remembered that venous thromboembolic disease including pulmonary embolism is more common in patients with an underlying malignancy (see section below), hence careful history taking is key in any initial venous thromboembolic assessment. Malignancy is also a clear risk factor for CTEPH [65].

\section{Other causes of tumoral PH}

Although rare, there are additional groups of cancer-related $\mathrm{PH}$, or apparent $\mathrm{PH}$, that are important to include within a section on tumoral PH. First, pulmonary vascular tumours may lie proximally within the main pulmonary artery vessels. For example, pulmonary artery angiosarcomas form an important although devastating differential diagnosis for patients presenting with apparent proximal venous thromboembolism or CTEPH [66], and are discussed in more detail below. Recognition even by experienced teams relies on advanced imaging including magnetic resonance and PET. Secondly, extrinsic compression by lymph nodes or mediastinal tumours, for example a metastatic thymic carcinoid tumour, can cause extrinsic compression mimicking stenosis of the pulmonary valve and apparent $\mathrm{PH}$ [67].

\section{Pulmonary artery angiosarcoma}

Sarcomas are rare tumours of mesenchymal origin that originate in bones or in soft tissues. Of these, angiosarcomas arise from endothelial cells and comprise $2 \%$ of all sarcomas [68]. Pulmonary artery angiosarcomas typically arise from the pulmonary trunk [69] and can metastasise to the lung and mediastinal lymph nodes, as well as through haematogenous dissemination. They are important albeit rare mimics of pulmonary thromboembolic disease.

Recognised associations with angiosarcoma include exposure to radiotherapy, genetic mutations including BRCA, neurofibromatosis type 1 and Kippel Trelawney syndrome, and patients with immunosuppression including HIV [68].

The clinical presentation of pulmonary artery angiosarcomas is nonspecific. The most common symptoms are dyspnoea, pleuritic chest pain, cough and haemoptysis [70].

CT findings include low attenuation filling defects, enhancement of the mass in the lumen of the artery and extravascular spread of the lesion [71]. Gadolinium-enhanced magnetic resonance imaging may help differentiate between thrombotic masses and vascular tumours: unlike thromboemboli, angiosarcomas exhibit heterogeneous enhancement with gadolinium [69]. The use of FDG-PET/CT can help differentiate malignant from benign pulmonary artery lesions [71].

Surgical treatment is preferred for patients with primary pulmonary angiosarcoma, and is only an option in the $50 \%$ presenting without metastatic disease [4]. Radical resection offers a median survival of $37 \pm 20$ months, compared to $11 \pm 3$ months following subtotal resection or debulking [72]. Without surgery, the mean survival is 1.5 months. Chemotherapy may improve survival, but evidence remains limited [72]. Inhibitors of angiogenesis may be more promising future therapies [73].

\section{Cancer treatment-related PH}

Several oncological treatments may promote the development of numerous subtypes of $\mathrm{PH}$ in patients with active or previous cancer. These aetiologies can be considered according to the $\mathrm{PH}$ classification, and of course may overlap with other causes of tumoral $\mathrm{PH}$ mentioned above.

\section{PAH induced by cancer therapies}

Of cancer therapies that can potentially induce PAH, the tyrosine kinase inhibitors (TKIs) are highlighted. TKIs include dasatanib [74], often used in chronic myeloid leukaemia therapy, and ponatinib, bosutinib and 
lapatinib, which are all used to treat several oncological conditions. Despite inhibiting relevant pathways including PDGF that might be expected to reverse PAH, these agents can cause direct pulmonary artery endothelial cell toxicity through the production of mitochondrial reactive oxygen species [75]. The PAH that arises usually resolves after discontinuation of the TKI, but persists in over a third of patients [76], and can be fatal in this setting, with irreversible remodelling reported requiring lung transplantation [77]. GUIGNABERT et al. [78] demonstrated a putative mechanism involving dasatinib-induced endothelial dysfunction and reactive oxygen species. TKI-induced PAH can unfortunately recur when other TKIs are used. Treatment with PAH-specific therapy is recommended for patients with right heart failure or persistent PAH after discontinuation of the TKI [75].

\section{PVOD induced by cancer therapies}

Several cancer treatment-related mechanisms may precipitate the onset of PVOD, with the common mechanism likely to relate to venous endothelial injury from cytotoxic chemotherapy or irradiation [79, 80]. PVOD is a rare, devastating form of $\mathrm{PAH}$, classified as group 1' $\mathrm{PH}$, characterised by widespread fibrous intimal proliferation of septal veins and preseptal venules also often with pulmonary capillary dilatation and proliferation [81] and substantial remodelling of the small pulmonary arteries [82]. Clinical features include marked oxygen desaturation and very low gas transfer, with a radiographic triad of interlobular septal thickening, pleural effusions and lymphadenopathy. PVOD has been described in patients receiving chemotherapy including gemcitabine [83] and mitomycin-C [79, 84-86], and also in several reports following bone marrow transplantation [80, 86-88]. Radiotherapy has been reported to induce PVOD, with a potential delay in onset for several years, for example following mantle irradiation for Hodgkin's lymphoma. Again, the mechanism is thought to relate to radiotherapy-induced venous endothelial damage [89]. It has been suggested that therapy-induced PVOD may be under-recognised by a recent series of patients who died with pulmonary graft versus host disease more than 1 year after allogeneic haematopoietic stem cell transplantation, where $34 \%$ had histological evidence for PVOD as well as $29 \%$ having evidence for obliterative bronchiolitis; in both cases far more than had been clinically recognised [90].

Recent advances have been made in the understanding of chemotherapy-induced PVOD. In 2002, GAGNADOUx et al. [85] described severe PVOD following surgery and perioperative mitomycin-C (MMC) chemotherapy for nonsmall cell lung carcinoma. More recently, PVOD cases from the French registry were identified whose anal carcinoma was treated with MMC, 5-flurouracil and radiotherapy 4 months prior to PVOD onset [2-12]. Baseline right heart catheterisation showed mean pulmonary artery pressure of $41 \mathrm{mmHg}$ and PVR of 11.2 (5.5-13.6) Wood units [36-63], with all patients in WHO functional class III or IV at presentation. Where tested, no patients had BMPR2 or EIF2AK4 mutations. Death followed in four out of the seven patients due to RV failure $(n=2)$, local tumour progression $(n=1)$ and severe pneumonia $(n=1)$. The same team developed a rat model of MMC-induced PVOD where intraperitoneal MMC led to severe PAH at day 21-35, major remodelling of small pulmonary veins with endothelial cell proliferation in the capillary bed. Interestingly, a perivenular accumulation of eosinophils was seen in this rat model [84]. In terms of signalling, a dose-dependent depletion of pulmonary GCN2 content and decreased smad1/5/8 signalling was measured, despite normal tissue BMPR2 levels. GCN2 is the protein coded by the recently described EIF2A2K gene whose function is depleted in heritable cases of PVOD, with the function of GCN2 likely to relate to antioxidant and anti-inflammatory properties [91]. These important observations by PERros et al. [84] have been highlighted by SAVALE et al. [92] in a case of severe PVOD in a 52-year-old woman with MMC, 5-flurouracil and local radiotherapy for anal cancer 6 months previously. She underwent stabilisation with intravenous diuretics and dobutamine, and initiation of an endothelin receptor antagonist. She was given intravenous methylprednisolone, given that perivenular inflammation was present in the rat MMC-induced PVOD model [84], with resulting radiographic improvement at 2 weeks (figure 5a) and clinical improvement that lasted a year.

However, she deteriorated further at that point requiring addition of a phosphodiesterase type-5 inhibitor, more steroids and ultimately successful lung transplantation. Pathologic assessment of explanted lungs confirmed the diagnosis of PVOD with arterial remodelling without plexiform lesions, fibrous venous occlusion and capillary congestion (figure 5), although no perivenular inflammation was noted [92].

\section{Preventative adjuncts}

Finally, in terms of chemotherapy- or radiotherapy-induced PAH/PVOD, there is an urgent clinical need to identify preventative adjuncts. Given the likely initial endothelial injury, cytoprotective approaches including amifostine, as well as steroids [92, 93], may be effective. Amifostine targets the endothelium, and is one of several cytoprotective agents developed to protect normal tissues from radiation and chemotherapy-induced damage. As an inactive prodrug, amifostine is dephosphorylated to an active thiol by alkaline phosphatase in normal endothelium, but not in acidic neoplastic tissues. This results in cytoprotective selectivity involving free radical scavenging, DNA protection and repair acceleration, and 

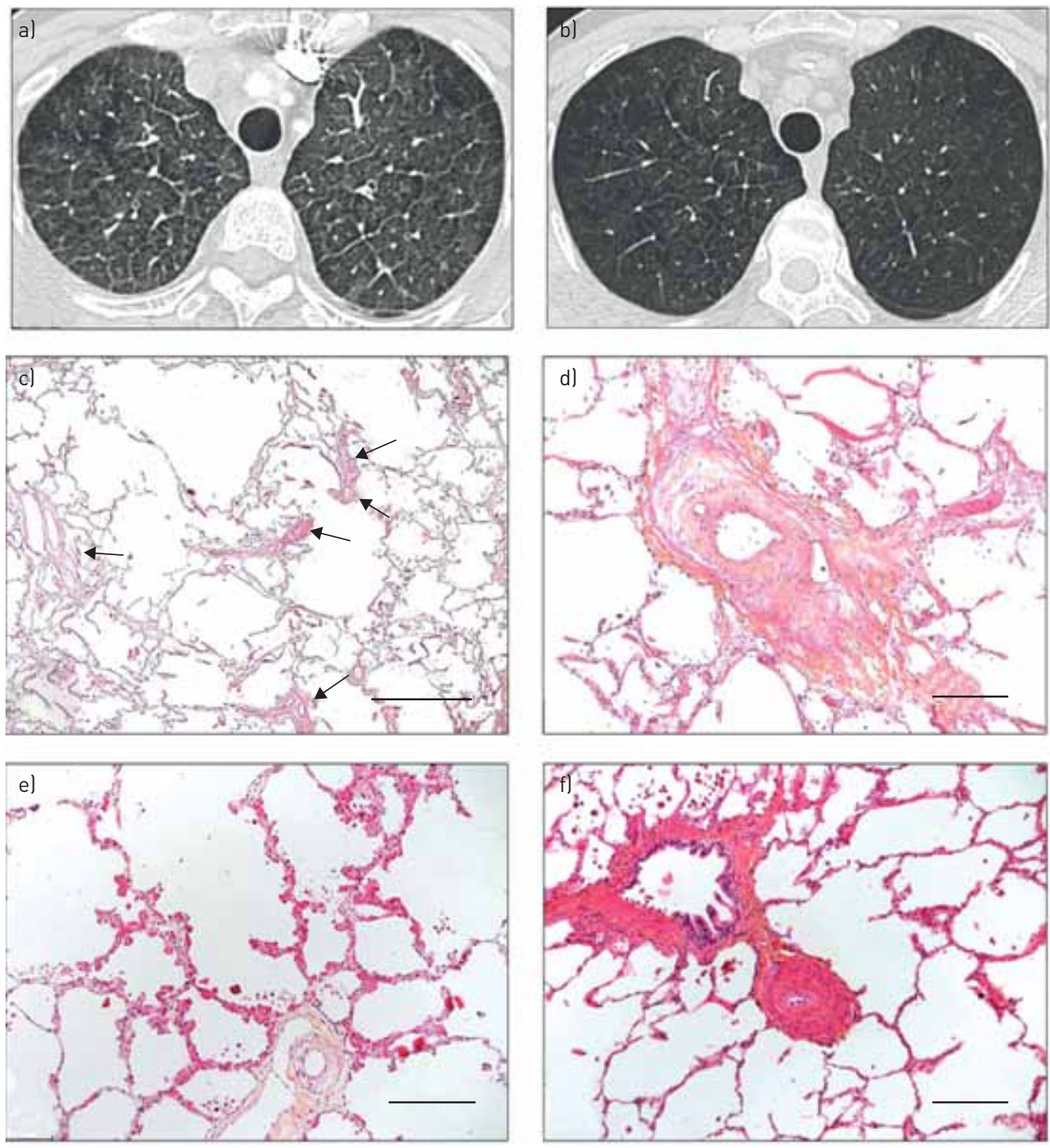

FIGURE 5 High-resolution computed tomography (HRCT) of the chest and lung histology (haematoxylin and eosin-saffron staining) from explanted lungs. a) HRCT at diagnosis showed septal lines and centrilobular ground-glass opacities suggestive of pulmonary veno-occlusive disease. b) Improvement of radiological abnormalities after 2 weeks of treatment including diuretics, dobutamine, endothelin receptor antagonist and high-dose corticosteroids. c) Overview of lung parenchyma displaying emphysematous changes and vasculopathy. Note the pulmonary vessels with narrowed lumina (arrows). d) Septal vein with pronounced intimal fibrosis and heavily narrowed lumen. e) Focal capillary congestion in the vicinity of a remodelled vein (bottom). f) Pulmonary artery (centre) with adjacent airway (top left) displaying medial hypertrophy and concentric intimal fibrosis. c) Scale bar $=1000 \mu \mathrm{m}$. d-f) Scale bar $=200 \mu \mathrm{m}$. Reproduced from [92].

induction of cellular hypoxia [94]. Perros et al. [84] showed that in the rat MMC-induced PVOD model, 2 weeks of amifostine co-treatment partially prevented the onset of MMC-induced PVOD, with improved haemodynamics and remodelling. Given the continuing clinical need for oncological therapies, although the incidence of therapy-induced PVOD and PAH is not yet known, over 30\% of patients had PVOD at autopsy following bone marrow transplantation [90]. The future use of cytoprotective therapies is, therefore, likely to be important.

\section{Group 2 PH induced by cancer therapies}

Chemotherapy and radiation can also cause left ventricular (LV) dysfunction [95], with the potential onset of group $2 \mathrm{PH}$. This leads to a form of cardiomyopathy, characterised by fall in LV ejection fraction, resulting in changes in biomarkers (troponin and BNP) and imaging, depending on the therapeutic agent and the stage of presentation. The fall in LV function may be irreversible or reversible depending on the agent, with the latter scenario highlighting the case for early recognition and monitoring [96]. Potential aetiologies include anthracyclines (doxorubicin, daunorubicin, epirubicin and idarubicin) [97], which are 
effective chemotherapeutic agents for solid and haematological tumours. In breast cancer, for example, doxorubicin and epirubicin are often used together. Anthracyclines are toxic to cardiac myocytes through intercalation into nuclear DNA, resulting in impaired protein synthesis and production of reactive oxygen species. This leads to inhibition of topoisomerase II thereby inhibiting DNA repair, ultimately leading to cell death [98], with resulting diastolic and systolic LV dysfunction [96]. Standard cardiovascular risk factors and additional therapies, including mediastinal irradiation, increase the risk of toxicity [96]. Alkylating agents (e.g. cyclophosphamide and melphalan) inhibit DNA transcription thereby altering protein synthesis. They are associated with LV dysfunction in up to $28 \%$ of patients, with a dose-related effect (at doses $>150 \mathrm{mg} \cdot \mathrm{kg}^{-1}$ ) seen soon after administration [99]. Additional cancer therapies that can cause LV dysfunction include VEGF inhibitors, (e.g. sunitinib and sorafenib) which are small molecule TKIs and nonselective VEGF inhibitors. These agents inhibit VEGF-mediated angiogenesis but also off target unwanted effects including those linked to LV dysfunction and heart failure [100]. Finally radiation-induced left heart disease is well recognised and may have onset years after exposure. Radiotherapy is associated with endothelial injury, valvular dysfunction, atherosclerosis, fibrosis and pericardial disease. Heart failure can occur as acute radiation myocarditis, but more commonly develops as a long-term consequence of fibrosis, leading to ventricular dysfunction or restrictive cardiomyopathy [101].

\section{Group 3 PH induced by cancer therapies}

In addition, certain cytotoxic chemotherapeutic agents (e.g. bleomycin) or frequent radiotherapy can cause pulmonary interstitial abnormalities and hypoxaemia [102], which may lead to group $3 \mathrm{PH}$. For example, cyclophosphamide can induce interstitial pneumonitis and fibrosis including cryptogenic organising pneumonia; total body irradiation can also lead to pneumonitis [103]. However, the more modern targeted conformal radiotherapeutic approaches significantly limit the risks of widespread lung damage and, therefore, $\mathrm{PH}$.

\section{Conclusions}

Tumoral PH principally includes tumour-related pulmonary microvascular conditions (PTE and PTTM), as well as tumour macroembolism and mediastinal or intravascular tumours (sarcomas) mimicking PH. In any patient with new-onset $\mathrm{PH}$ of unknown origin, the presence of an underlying malignancy should also be considered. In addition, the long-term pulmonary vascular effects of cancer therapies (importantly including PVOD) is a growing area and should be considered in the differential diagnosis of a cancer patient presenting with breathlessness or $\mathrm{PH}$. Autopsy studies from cancer patients indicate that the finding of tumour emboli within small pulmonary vessels is not uncommon. PTE is the term used for simple tumour cell emboli; PTTM develops in some patients with more extensive remodelling, with similarities and differences to PAH in terms of vessel architecture and signalling pathways. It is not well understood why some patients with cancer harbour asymptomatic tumour emboli, whereas others develop more extensive remodelling with severe changes in PTTM leading to progressive PH and severe fatal RV failure; future genetic studies will be of interest. The diagnosis of PTE and PTTM can be difficult ante mortem, but with increasing recognition of the condition, therapeutic strategies are marginally improving the outlook. Although prognosis of tumoral PH remains very poor, temporary response in PTE/PTTM to chemotherapeutic agents, antiproliferative approaches and pulmonary vasodilators is described. Further understanding of these malignant causes of $\mathrm{PH}$ is clearly needed.

Small nests of carcinomatous cells lodge in pulmonary vessels, including small pre-capillary arteries (via haematogenous spread) and veins and lymphatics (by lymphatic invasion). Tumour cell and endothelial cell interaction initiate clot formation, and release further cytokines including VEGF and PDGF. This initiates macrophage recruitment and intimal (endothelial cell and non-endothelial like, i.e. myofibroblastic) proliferation. Tissue factor also upregulates VEGF expression on tumour cells, which is angiogenic to intimal cells. PDGF-A and -B are expressed on tumour cells and anti-phospho-PDGFR- $\alpha$ on vascular endothelial cells and gastric carcinoma cells, indicating PDGF signalling activation through autocrine and paracrine mechanisms. The cytokine and adhesive protein osteopontin is expressed on tumour cells in PTTM, and is likely to be a key driver for intimal cell growth. Perivascular CD68-positive macrophages are noted and also reside within intimal layers. Macrophages also stain for CD44, the adhesion molecule, which interacts with osteopontin to induce chemotaxis of T-cells and macrophages, propagation of local inflammation and intimal proliferation (through other known macrophage-derived pro-proliferative factors including interleukin-6). Direct contact with tumour nests is not universal in all vessels where remodelling is present.

Conflict of interest: L.C. Price reports personal fees from Actelion/Johnson and Johnson and has received an educational grant from GSK Pharmaceuticals in the last 2 years. M.J. Seckl has nothing to disclose. P. Dorfmüller reports personal fees from MSD, Actelion and Roche, outside the submitted work. S.J. Wort reports grants and personal fees from Actelion and Bayer, and personal fees from GSK and MSD, outside the submitted work. 


\section{References}

1 Roberts KE, Hamele-Bena D, Saqi A, et al. Pulmonary tumor embolism: a review of the literature. Am J Med 2003; 115: 228-232.

2 Kane RD, Hawkins HK, Miller JA, et al. Microscopic pulmonary tumor emboli associated with dyspnea. Cancer 1975; 36: 1473-1482.

3 Bagshawe KD, Brooks WD. Subacute pulmonary hypertension due to chorionepithelioma. Lancet 1959; 1: 653-658.

4 Shields DJ, Edwards WD. Pulmonary hypertension attributable to neoplastic emboli: an autopsy study of 20 cases and a review of literature. Cardiovasc Pathol 1992; 1: 279-287.

5 Winterbauer RH, Elfenbein IB, Ball WC Jr. Incidence and clinical significance of tumor embolization to the lungs. Am J Med 1968; 45: 271-290.

6 Soares FA, Pinto AP, Landell GA, et al. Pulmonary tumor embolism to arterial vessels and carcinomatous lymphangitis. A comparative clinicopathological study. Arch Pathol Lab Med 1993; 117: 827-831.

7 Wilson K, Guardino J, Shapira O. Pulmonary tumor embolism as a presenting feature of cavoatrial hepatocellular carcinoma. Chest 2001; 119: 657-658.

8 Schriner RW, Ryu JH, Edwards WD. Microscopic pulmonary tumor embolism causing subacute cor pulmonale: a difficult antemortem diagnosis. Mayo Clin Proc 1991; 66: 143-148.

9 Yutani C, Imakita M, Ishibashi-Ueda H, et al. Pulmonary hypertension due to tumor emboli: a report of three autopsy cases with morphological correlations to radiological findings. Acta Pathol Jpn 1993; 43: 135-141.

10 Seckl MJ, Rustin GJ, Newlands ES, et al. Pulmonary embolism, pulmonary hypertension, and choriocarcinoma. Lancet 1991; 338: 1313-1315.

11 Goldhaber SZ, Dricker E, Buring JE, et al. Clinical suspicion of autopsy-proven thrombotic and tumor pulmonary embolism in cancer patients. Am Heart J 1987; 114: 1432-1435.

12 von Herbay A, Illes A, Waldherr R, et al. Pulmonary tumor thrombotic microangiopathy with pulmonary hypertension. Cancer 1990; 66: 587-592.

13 Pinckard JK, Wick MR. Tumor-related thrombotic pulmonary microangiopathy: review of pathologic findings and pathophysiologic mechanisms. Ann Diagn Pathol 2000; 4: 154-157.

14 Chinen K, Tokuda Y, Fujiwara M, et al. Pulmonary tumor thrombotic microangiopathy in patients with gastric carcinoma: an analysis of 6 autopsy cases and review of the literature. Pathol Res Pract 2010; 206: 682-689.

15 Carter CA, Scicinski JJ, Lybeck HE, et al. Pulmonary tumor thrombotic microangiopathy: a new paraneoplastic syndrome? Case Rep Oncol 2016; 9: 246-248.

16 Uga S, Ikeda S, Matsukage S, et al. An autopsy case of acute cor pulmonale and paradoxical systemic embolism due to tumour cell microemboli in a patient with breast cancer. BMJ Case Rep 2012; 2012: bcr2012006682.

17 Buser M, Felizeter-Kessler M, Lenggenhager D, et al. Rapidly progressive pulmonary hypertension in a patient with pulmonary tumor thrombotic microangiopathy. Am J Respir Crit Care Med 2015; 191: 711-712.

18 Gru AA, Pai RK, Roma AA. Pulmonary tumor thrombotic microangiopathy in patients with low-grade ovarian serous neoplasm: a clinicopathologic review of 2 cases of a previously unknown association. Int J Gynecol Pathol 2012; 31: 438-442.

19 Tanaka K, Nakasya A, Miyazaki M, et al. [A case of hepatocellular carcinoma with respiratory failure caused by widespread tumor microemboli]. Fukuoka Igaku Zasshi 2011; 102: 298-302.

20 Malani AK, Gupta C, Kutty AV, et al. Pulmonary tumor thrombotic microangiopathy from metastatic gallbladder carcinoma: an unusual cause of severe pulmonary hypertension. Dig Dis Sci 2007; 52: 555-557.

21 Kumar N, Price LC, Montero MA, et al. Pulmonary tumour thrombotic microangiopathy: unclassifiable pulmonary hypertension? Eur Respir J 2015; 46: 1214-1217.

22 von Herbay A, Maiwald M, Ditton HJ, et al. Histology of intestinal Whipple's disease revisited. A study of 48 patients. Virchows Arch 1996; 429: 335-343.

23 Uruga H, Fujii T, Kurosaki A, et al. Pulmonary tumor thrombotic microangiopathy: a clinical analysis of 30 autopsy cases. Intern Med 2013; 52: 1317-1323.

24 Okubo Y, Wakayama M, Kitahara K, et al. Pulmonary tumor thrombotic microangiopathy induced by gastric carcinoma: morphometric and immunohistochemical analysis of six autopsy cases. Diagn Pathol $2011 ; 6: 27$

25 Minatsuki S, Miura I, Yao A, et al. Platelet-derived growth factor receptor-tyrosine kinase inhibitor, imatinib, is effective for treating pulmonary hypertension induced by pulmonary tumor thrombotic microangiopathy. Int Heart J 2015; 56: 245-248.

26 Price LC, Wells AU, Wort SJ. Pulmonary tumour thrombotic microangiopathy. Curr Opin Pulm Med 2016; 22: 421-428.

27 Takahashi F, Kumasaka T, Nagaoka T, et al. Osteopontin expression in pulmonary tumor thrombotic microangiopathy caused by gastric carcinoma. Pathol Int 2009; 59: 752-756.

28 Higashi A, Dohi Y, Uraoka N, et al. The Potential role of inflammation associated with interaction between osteopontin and CD44 in a case of pulmonary tumor thrombotic microangiopathy caused by breast cancer. Intern Med 2015; 54: 2877-2880.

29 Khan SA, Cook AC, Kappil M, et al. Enhanced cell surface CD44 variant (v6, v9) expression by osteopontin in breast cancer epithelial cells facilitates tumor cell migration: novel post-transcriptional, post-translational regulation. Clin Exp Metastasis 2005; 22: 663-673.

30 Hashimoto-Kataoka T, Hosen N, Sonobe T, et al. Interleukin-6/interleukin-21 signaling axis is critical in the pathogenesis of pulmonary arterial hypertension. Proc Natl Acad Sci USA 2015; 112: E2677-E2686.

31 Godbole R, Saggar R, Zider A, et al. Insights on pulmonary tumor thrombotic microangiopathy: a seven-patient case series. Pulm Circ 2017; 7: 813-820.

32 Dorfmuller P, Gunther S, Ghigna MR, et al. Microvascular disease in chronic thromboembolic pulmonary hypertension: a role for pulmonary veins and systemic vasculature. Eur Respir J 2014; 44: 1275-1288.

33 Caine GJ, Stonelake PS, Lip GY, et al. The hypercoagulable state of malignancy: pathogenesis and current debate. Neoplasia 2002; 4: 465-473.

34 Wahrenbrock M, Borsig L, Le D, et al. Selectin-mucin interactions as a probable molecular explanation for the association of Trousseau syndrome with mucinous adenocarcinomas. J Clin Invest 2003; 112: 853-862. 
Gainza E, Fernandez S, Martinez D, et al. Pulmonary tumor thrombotic microangiopathy: report of 3 cases and review of the literature. Medicine (Baltimore) 2014; 93: 359-363.

Ho AL, Szulakowski P, Mohamid WH. The diagnostic challenge of pulmonary tumour thrombotic microangiopathy as a presentation for metastatic gastric cancer: a case report and review of the literature. BMC Cancer 2015; 15: 450.

Miyano S, Izumi S, Takeda Y, et al. Pulmonary tumor thrombotic microangiopathy. J Clin Oncol 2007; 25: 597-599. Simonneau G, Galie N, Rubin LJ, et al. Clinical classification of pulmonary hypertension. J Am Coll Cardiol 2004; 43: 12 Suppl S, 5S-12S.

Galie N, Humbert M, Vachiery J-L, et al. 2015 ESC/ERS Guidelines for the diagnosis and treatment of pulmonary hypertension. The Joint Task Force for the Diagnosis and Treatment of Pulmonary Hypertension of the European Society of Cardiology (ESC) and the European Respiratory Society (ERS). Eur Respir J 2015; 46: 903-975.

Kayatani H, Matsuo K, Ueda Y, et al. Pulmonary tumor thrombotic microangiopathy diagnosed antemortem and treated with combination chemotherapy. Intern Med 2012; 51: 2767-2770.

Ogawa A, Yamadori I, Matsubara O, et al. Pulmonary tumor thrombotic microangiopathy with circulatory failure treated with imatinib. Intern Med 2013; 52: 1927-1930.

116: 131-134.

Montani D, Achouh L, Dorfmuller P, et al. Pulmonary veno-occlusive disease: clinical, functional, radiologic, and hemodynamic characteristics and outcome of 24 cases confirmed by histology. Medicine (Baltimore) 2008; 87: 220-233.

Seferian A, Helal B, Jais X, et al. Ventilation/perfusion lung scan in pulmonary veno-occlusive disease. Eur Respir J 2012; 40: 75-83.

Godbole R, Ghatol A, Betancourt J, et al. Pulmonary tumor thrombotic microangiopathy: clinical, radiologic, and histologic correlation. J Clin Imaging Sci 2015; 5: 44

Franquet T, Gimenez A, Prats R, et al. Thrombotic microangiopathy of pulmonary tumors: a vascular cause of tree-in-bud pattern on CT. AJR Am J Roentgenol 2002; 179: 897-899.

Kitamura A, Nishimura N, Jinta T, et al. A case of pulmonary tumor thrombotic microangiopathy diagnosed by transbronchial lung biopsy and treated with chemotherapy and long-term oxygen and anticoagulation therapies. Case Rep Pulmonol 2013; 2013: 259080.

The Nucl Med 2009; 34: 175-177.

Carrier M, Lazo-Langner A, Shivakumar S, et al. Screening for occult cancer in unprovoked venous thromboembolism. N Engl J Med 2015; 373: 697-704.

Hutchinson JC, Fulcher JW, Hanna J, et al. Pulmonary tumor thrombotic microangiopathy: case report and review of literature. Am J Forensic Med Pathol 2018; 39: 56-60.

Bhuvaneswaran JS, Venkitachalam CG, Sandhyamani S. Pulmonary wedge aspiration cytology in the diagnosis of recurrent tumour embolism causing pulmonary arterial hypertension. Int J Cardiol 1993; 39: 209-212.

A, Ott K, Weber WA, et al. FDG PET imaging of locally advanced gastric carcinomas: correlation with endoscopic and histopathological findings. Eur J Nucl Med Mol Imaging 2003; 30: 288-295.

Keenan NG, Nicholson AG, Oldershaw PJ. Fatal acute pulmonary hypertension caused by pulmonary tumour thrombotic microangiopathy. Int J Cardiol 2008; 124: el1-e13.

Simonneau G, Robbins IM, Beghetti M, et al. Updated clinical classification of pulmonary hypertension. J Am Coll Cardiol 2009; 54: 1 Suppl, S43-S54.

Savage P, Roddie M, Seckl MJ. A 28-year-old woman with a pulmonary embolus. Lancet 1998; 352: 30. inadequate response to established therapy. Am J Respir Crit Care Med 2010; 182: 1171-1177.

Hoeper MM, Barst RJ, Bourge RC, et al. Imatinib mesylate as add-on therapy for pulmonary arterial hypertension: results of the randomized IMPRES study. Circulation 2013; 127: 1128-1138.

Higo K, Kubota K, Takeda A, et al. Successful antemortem diagnosis and treatment of pulmonary tumor thrombotic microangiopathy. Intern Med 2014; 53: 2595-2599.

Geschwind JF, Dagli MS, Vogel-Claussen J, et al. Metastatic breast carcinoma presenting as a large pulmonary embolus: case report and review of the literature. Am J Clin Oncol 2003; 26: 89-91.

Jakel J, Ramaswamy A, Kohler U, et al. Massive pulmonary tumor microembolism from a hepatocellular carcinoma. Pathol Res Pract 2006; 202: 395-399.

Perroni D, Grecchi GL, La Ciura P, et al. Right ventricular metastasis from choriocarcinoma: report of a rare case and review of the literature. Eur J Surg Oncol 1993; 19: 378-381.

Bozaci EA, Taskin S, Gurkan O, et al. Intracavitary cardiac metastasis and pulmonary tumor emboli of choriocarcinoma: the first case diagnosed and treated without surgical intervention. Gynecol Oncol 2005; 99: 753-756.

Han GH, Kwon DY, Ulak R, et al. Right ventricular metastatic tumor from a primary carcinoma of uterine cervix: a cause of pulmonary embolism. Obstet Gynecol Sci 2017; 60: 129-132.

Jann $\mathrm{H}$, Wertenbruch T, Pape U, et al. A matter of the heart: myocardial metastases in neuroendocrine tumors. Horm Metab Res 2010; 42: 967-976.

Kim NH, Lang IM. Risk factors for chronic thromboembolic pulmonary hypertension. Eur Respir Rev 2012; 21: $27-31$.

Govender D, Pillay SV. Right pulmonary artery sarcoma. Pathology 2001; 33: 243-245. metastatic thymic carcinoid tumor. Int J Card Imaging 1996; 12: 61-63.

Young RJ, Brown NJ, Reed MW, et al. Angiosarcoma. Lancet Oncol 2010; 11: 983-991.

Huo L, Lai S, Gladish G, et al. Pulmonary artery angiosarcoma: a clinicopathologic and radiological correlation. Ann Diagn Pathol 2005; 9: 209-214.

marais $\mathrm{P}$, Laskine $\mathrm{M}$, Caporuscio $\mathrm{C}$. Primary pulmonary artery angiosarcoma mimicking pulmonary embolism in a 66-year-old man with dyspnea. CMAJ 2016; 188: E509-E512. 
101 Prosnitz

102 Abid SH, Malhotra V, Perry MC. Radiation-induced and chemotherapy-induced pulmonary injury. Curr Opin

Oncol 2001; 13: 242-248.

Lee EJ, Moon SH, Choi JY, et al. Usefulness of fluorodeoxyglucose positron emission tomography in malignancy of pulmonary artery mimicking pulmonary embolism. ANZ J Surg 2013; 83: 342-347.

Blackmon SH, Rice DC, Correa AM, et al. Management of primary pulmonary artery sarcomas. Ann Thorac Surg 2009; 87: 977-984.

Azzariti A, Porcelli L, Mangia A, et al. Irradiation-induced angiosarcoma and anti-angiogenic therapy: a therapeutic hope? Exp Cell Res 2014; 321: 240-247.

Montani D, Bergot E, Gunther S, et al. Pulmonary arterial hypertension in patients treated by dasatinib. Circulation 2012; 125: 2128-2137.

Weatherald J, Chaumais MC, Montani D. Pulmonary arterial hypertension induced by tyrosine kinase inhibitors. Curr Opin Pulm Med 2017; 23: 392-397.

Weatherald J, Chaumais MC, Savale L, et al. Long-term outcomes of dasatinib-induced pulmonary arterial hypertension: a population-based study. Eur Respir J 2017; 50: 1700217.

Daccord C, Letovanec I, Yerly P, et al. First histopathological evidence of irreversible pulmonary vascular disease in dasatinib-induced pulmonary arterial hypertension. Eur Respir J 2018; 51: 1701694.

Guignabert C, Phan C, Seferian A, et al. Dasatinib induces lung vascular toxicity and predisposes to pulmonary hypertension. J Clin Invest 2016; 126: 3207-3218.

Joselson R, Warnock M. Pulmonary veno-occlusive disease after chemotherapy. Hum Pathol 1983; 14: 88-91.

Bunte MC, Patnaik MM, Pritzker MR, et al. Pulmonary veno-occlusive disease following hematopoietic stem cell transplantation: a rare model of endothelial dysfunction. Bone Marrow Transplant 2008; 41: 677-686.

Montani D, Price LC, Dorfmuller P, et al. Pulmonary veno-occlusive disease. Eur Respir J 2009; 33: $189-200$.

( 647-655.

Turco C, Jary M, Kim S, et al. Gemcitabine-induced pulmonary toxicity: a case report of pulmonary veno-occlusive disease. Clin Med Insights Oncol 2015; 9: 75-79.

human disease and animal models. Circulation 2015; 132: 834-847.

Gagnadoux F, Capron F, Lebeau B. Pulmonary veno-occlusive disease after neoadjuvant mitomycin chemotherapy and surgery for lung carcinoma. Lung Cancer 2002; 36: 213-215.

Seguchi M, Hirabayashi N, Fujii Y, et al. Pulmonary hypertension associated with pulmonary occlusive vasculopathy after allogeneic bone marrow transplantation. Transplantation 2000; 69: 177-179. transplantation. Thorax 1984; 39: 956-957.

Williams LM, Fussell S, Veith RW, et al. Pulmonary veno-occlusive disease in an adult following bone marrow transplantation. Case report and review of the literature. Chest 1996; 109: 1388-1391.

Kramer MR, Estenne M, Berkman N, et al. Radiation-induced pulmonary veno-occlusive disease. Chest 1993; 104: $1282-1284$.

Gazourian L, Spring L, Meserve E, et al. Pulmonary clinicopathological correlation after allogeneic hematopoietic stem cell transplantation: an autopsy series. Biol Blood Marrow Transplant 2017; 23: 1767-1772.

Wilson GJ, Bunpo P, Cundiff JK, et al. The eukaryotic initiation factor 2 kinase GCN2 protects against hepatotoxicity during asparaginase treatment. Am J Physiol Endocrinol Metab 2013; 305: E1124-E1133.

Savale L, Chaumais MC, Dorfmuller P, et al. Lung transplantation for mitomycin-induced pulmonary veno-occlusive disease. Presse Med 2017; 46: 1223-1225. allogeneic bone marrow transplantation for acute myeloid leukemia. Intern Med 2012; 51: 195-198.

Kouvaris JR, Kouloulias VE, Vlahos LJ. Amifostine: the first selective-target and broad-spectrum radioprotector Oncologist 2007; 12: 738-747. Nat Rev Clin Oncol 2013; 10: 697-710. definitions, pathophysiology, risk factors, and imaging. Circ Heart Fail 2016; 9: e002661.

Shan K, Lincoff AM, Young JB. Anthracycline-induced cardiotoxicity. Ann Intern Med 1996; 125: 47-58.

Lyu YL, Kerrigan JE, Lin CP, et al. Topoisomerase IIbeta mediated DNA double-strand breaks: implications in doxorubicin cardiotoxicity and prevention by dexrazoxane. Cancer Res 2007; 67: 8839-8846.

, Antin JH, Plappert MT, et al. Cyclophosphamide cardiotoxicity in bone marrow transplantation: a prospective evaluation of new dosing regimens. J Clin Oncol 1991; 9: 1215-1223.

Groarke JD, Choueiri TK, Slosky D, et al. Recognizing and managing left ventricular dysfunction associated with therapeutic inhibition of the vascular endothelial growth factor signaling pathway. Curr Treat Options Cardiovasc Med 2014; 16: 335. 